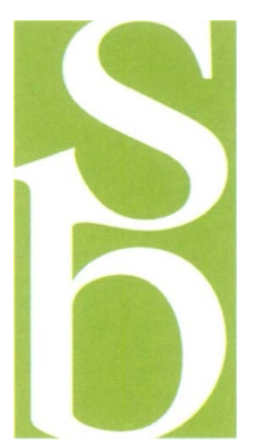

\title{
The connective tissue connection
}

No fewer than three of the reports in this issue concern the matrix metalloproteinases $\left(\mathrm{MMPs}^{1-3}\right)$, zinc-dependent extracellular proteinases which include collagenase, and more papers have recently appeared, or are about to appear, elsewhere ${ }^{4-6}$. As Tom Blundell discusses in a News \& Views article on pages $73-75$, these papers mark a major step in the quest for agents with which to treat diseases like arthritis and cancer.

The interest of these structures is two-fold. First, they reveal at once the common fold and some of the structural differences within this protein family, as well as their relationship to other zinc endopeptidases such as astacin, thermolysin and elastase. All these proteins have been grouped into a common superfamily, the metzincins. Collectively the MMPs are involved in extracellular degradation of the protein components of connective tissue. Under normal circumstances the enzymes are important in the tissue remodelling which occurs during embryogenesis, development and wound healing and possibly cell migration and angiogenesis as well.

There are also medical implications: enzymes of the MMP family are associated with diseases like rheumatoid arthritis, osteoarthritis, periodontitis and tumour metastasis. It may not be surprising that all but one of these new structures come from the laboratories of major pharmaceutical companies ${ }^{1-3,5,6}$, many of which are investing considerable effort in developing synthetic inhibitors of these enzymes as drugs against cancer and arthritis.

The present treatments for these conditions leave much to be desired. For rheumatoid arthritis anti-inflammatory agents such as cyclooxygenase inhibitors and steroids, which are used to treat the symptoms of the disease, and disease modifying agents, such as gold and methotrexate, can all cause extremely unpleasant side-effects. New treatments are much sought after, and inhibitors of collagenase and other MMPs are potentially attractive.

Candidate compounds are not lacking, and the structures of MMP catalytic domains should allow their refinement into even more specific inhibitors. Of course, these efforts will not be plain sailing. Designing a drug effective at femtomolar concentrations in vitro is merely the beginning of the task. Toxicity, bioavailability and active half-life are all hurdles at which a compound may afterwards stumble. But starting with one with high affinity in vitro may at least allow the tradeoffs necessary to develop a drug which is effective and acceptable. Indeed, at least one company now claims to have produced an orally available collagenase inhibitor active against cartilage degradation in vivo; clinical trials of the drug are imminent.

Yet the details of the role of MMPs in disease are still unclear. Some pharmaceutical companies are therefore wary of emphasizing any one of these enzymes in their drug development programmes, believing that more research into the normal role of the enzyme is needed. The advent of specific inhibitors may also, of course, advance this goal, by allowing the function of the different enzymes to be dissected.

But why the sudden flurry of MMP structures? The explanation seems to be from the use of recombinant DNA technology to both isolate the MMP genes and purify large amounts of the proteins, and in particular the catalytic domains, without the problems of scarcity and autoproteolysis that complicate purification of the native enzymes.

Several scientists have suggested that this rash of structures may have also been induced 
in part by panic stemming from a rumour that one of the groups involved was about to publish its results. Clearly, the pressure on commercial concerns to gain maximum benefit from their own research is intense: the cost of $\mathrm{R} \& \mathrm{D}$ in the major pharmaceutical companies is escalating to levels that may not be indefinitely sustainable.

What now emerges from the research is that the global fold consists of a five-stranded $\beta$ sheet flanked by three $\alpha$-helices, one of which contains the sequence His-Glu- $x$-His, with two of these histidines acting as ligands to the catalytic zinc. The active site is a groove, formed between one of the strands of $B$-sheet, one of the helices and a loop, on the surface of the roughly spherical proteins. Details of the bound inhibitors and of the local topology in the vicinity of the active sites of the various enzymes will fuel design of enzymespecific drugs.

Isolated catalytic domains of the MMPs have activities very similar to those of the native proteins, although they are not always identical; in the case of collagenase, for example, the catalytic domain is active against peptides, but not against collagen itself, the in vivo substrate. Although the major features of the MMP class of enzymes are now clear, it must be hoped that the structures of the intact proteins will throw light on crucial variations of specificity such as this.

\section{Partners in folding}

Knowledge of protein folding pathways is becoming ever more detailed. Two papers in our previous issue, for example, described the structure of partially folded proteins - highly ordered 'molten globules' - that may provide models for late folding intermediates ${ }^{7,8}$. As this knowledge increases, the interest in how it can be integrated into what is known of the folding process in vivo grows.

In the cell, nascent polypeptide chains or unfolded proteins are very likely to aggregate, and their (re)folding is assisted by a class of proteins known as chaperones, which prevent aggregation and suppress the formation of non-productive folding intermediates.

Chaperones of the heat-shock protein 70 (hsp70) class interact with nascent polypeptides, and are involved in a number of other folding-related events. These proteins bind and release proteins in an ATP-dependent manner. The low intrinsic ATPase activity of the hsp70 homologue in Escherichia coli, DnaK, is controlled by two other proteins: DnaJ and the nucleotide exchange factor GrpE. The latter mediates the dissociation of DnaK-bound nucleotides and thus the release of bound substrate and chaperone recycling.

The next step is clearly to understand the interactions of DnaK, GrpE and DnaJ at the molecular level, and the paper from Buchberger et al. in this issue $e^{9}$ shows one way in which this might be done. Using a series of binding, ATPase and folding assays with various recombinant and mutant forms of DnaK, Buchberger et al. show that a loop in close proximity to the N-terminal ATP binding site interacts with GrpE. This fits what is known of GrpE's role as the nucleotide exchange factor.

This loop is isolated on the surface of the DnaK protein, and is conserved in all members of the hsp70 family. It is absent in structurally related proteins, such as actin and hexokinase, however, suggesting an hsp70-specific function. The authors point out that this degree of conservation suggests that the eukaryotic hsp70 proteins are also regulated by GrpE homologues, and that such homologues may already have been found.

1. Borkakoti, N. etal. Nature struct. Biol. 1, 106-110(1994).

2. Gooley, P.R. etal. Nature struct. Biol. 1, 111-118(1994).

3. Stams, T. et al. Nature struct. Biol. 1, 119-123 (1994).

4. Bode, W. et al. EMBO J. in the press (1994).

5. Lovejoy, B. et al. Science in the press (1994).

6. Spurlino, J.C. et al. Proteins in the press (1994).

7. Redfield, R., Smith, A.G., and Dobson, C.M. Nature struct. Biol. 1, 23-29 (1994)

8. Feng, Y., Sligar, S.G. \& Wand, A.J. Nature struct. Biol. 1, 30-35 (1994).

9. Buchberger, A., Schröder, H., Büttner, M., Valencia, A. and Bukau, B. Nature struct. Biol. 1, 95-101 (1994).

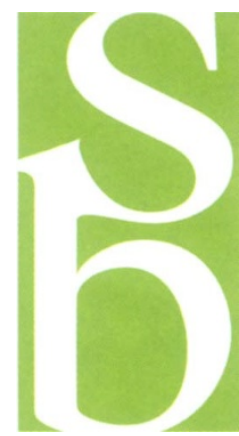

